\title{
marketplace
}

\section{Avoid litigation}

If a patient does not understand the treatments you are suggesting or why they need them, they will be unable to give informed consent. To avoid potential litigation as a result of this, Admor, in association with Dental Protection, has developed a series of Treatment Consent protocols to ensure that patients are fully informed about their treatment plans. In particular, the NCR (no carbon required) pad designed for crowns and bridges provides a variety of advice and information to ensure the dentist gains consent in an appropriate manner and that the patient has access to information needed to understand exactly what they are consenting to.

A set of five sheets provides information and guidance on commonly asked questions such as what crowns and bridges are and who needs them. There is also a two set consultation form (a copy each for patient and dentist) on which details of the consultation can be recorded, with space for a patient signature.

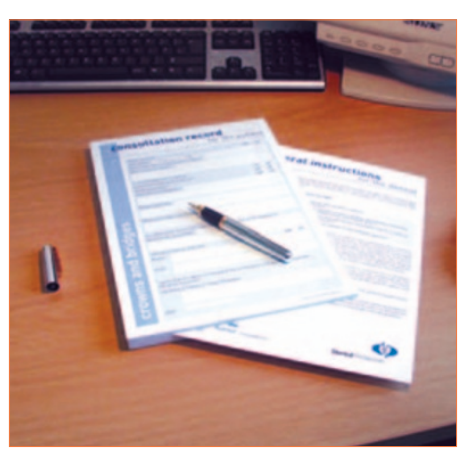

The dentist can file their copy with patient records or scan it on to a computer-based record, and the patient copy has simple, easy to follow advice on the reverse to give further detailed information to the patient and pre and post treatment advice.

For more information visit www.admor.co.uk or contact 01243553078

\section{Enjoying the view}

Designer Dental has launched an additional range to their poster collection with a choice of ceiling posters. These posters are in full colour and come in three sizes with six different designs, are bright, vibrant and cover a range of scenic and world features. They have also been designed to suit most practice colour schemes so they can easily blend into surgery surroundings. The posters can be viewed and ordered via their website at www. designerdental.co.uk.

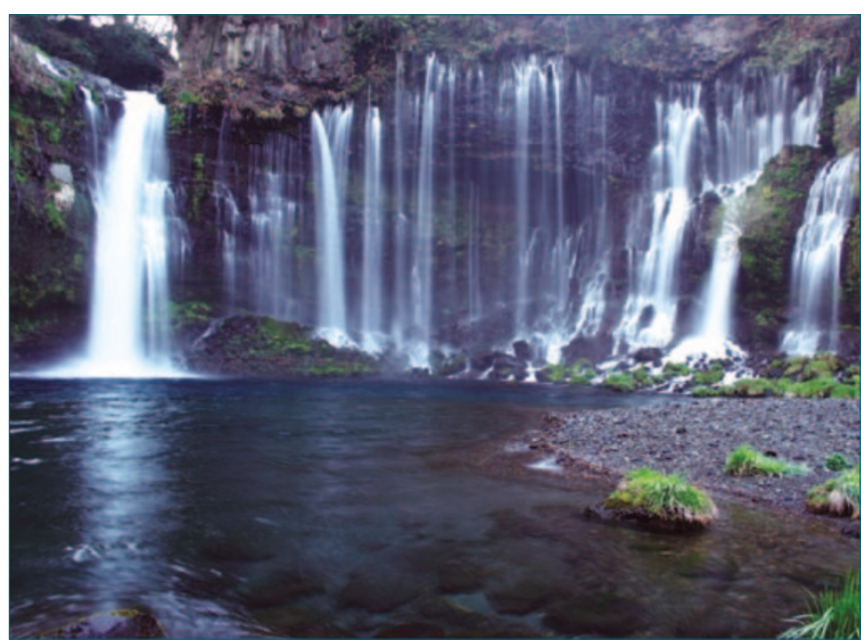

\section{Automatic dispenser}

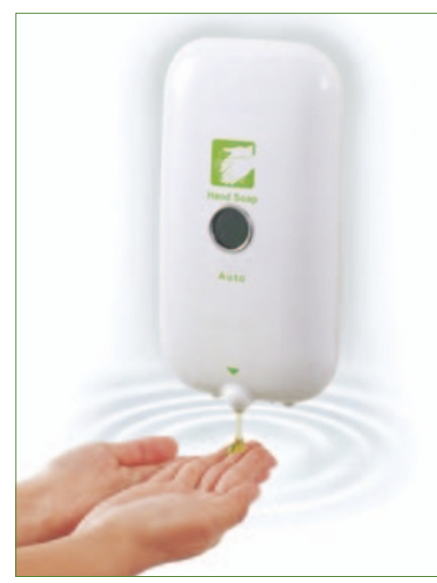

Alkapharm, specialists in a range of professional products to aid the control of cross infection within the dental surgery, have introduced the new Sanishot range of 'touchless' hand soap and hand disinfectant dispensers. The products detect the presence of hands then automatically dispense the optimum amount of hand soap or disinfectant into the hands. For information visit www.alkapharm.co.uk.

\section{Practical workwear solutions}

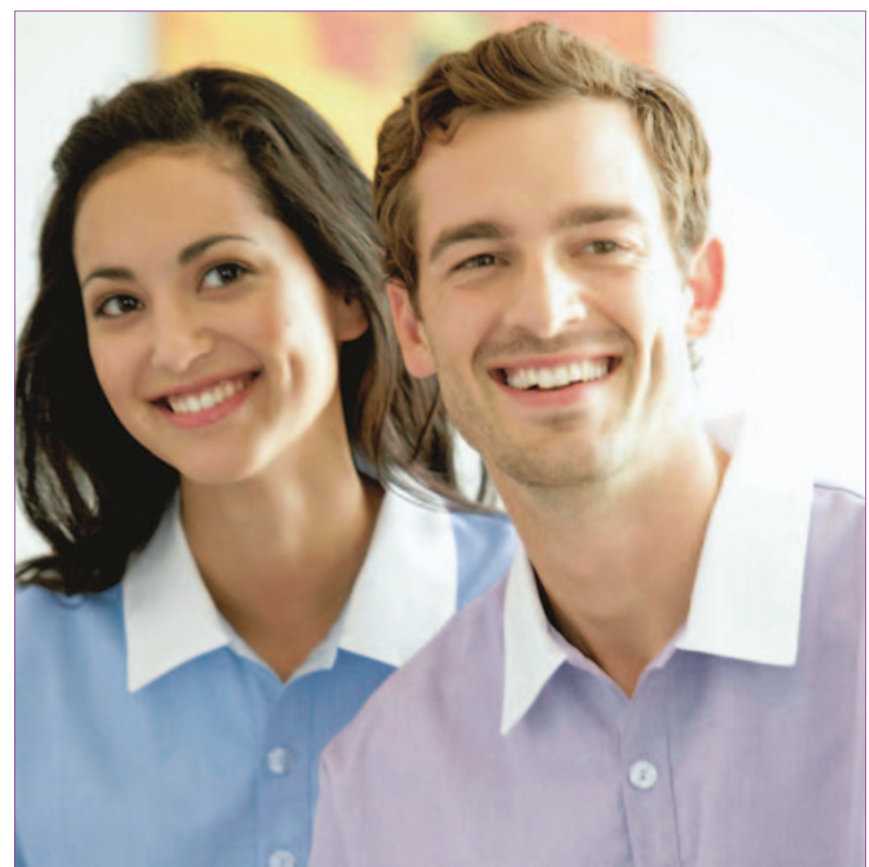

Hejco's Oxford Collection is presented in a range of attractive colour combinations to create a soft, pleasantly relaxed impression. This range of blouses and unisex shirts is designed to complement all Hejco trousers, allowing you to choose the most suitable combination for your needs. The Oxford range's comfortable, loose fitting design permits unrestricted movement but also ensures that nothing is revealed when you bend or stretch!

The Twill Range of work wear is fashioned for the hustle and bustle of life in busy modern practice. Its loose fitting, robust styling is designed to cope with the unexpected as well as the day to day routine of practice life. Although manufactured in a stronger fabric, it still hangs well from the body and gives a flattering fit. Matching trousers are also available for a coordinated look. The range is a practical workwear solution, furnished with a number of pockets which includes a specially designed, divided breast pocket ideal for keeping pens organised. For further information call 01634878787. 